\title{
A study of Pc-5 ULF oscillations
}

\author{
M. K. Hudson ${ }^{1}$, R. E. Denton ${ }^{1}$, M. R. Lessard ${ }^{2}$, E. G. Miftakhova ${ }^{1}$, and R. R. Anderson ${ }^{3}$ \\ ${ }^{1}$ Department of Physics and Astronomy, Dartmouth College, Hanover, NH 03755, USA \\ ${ }^{2}$ Thayer School of Engineering, Dartmouth College, Hanover, NH 03755, USA \\ ${ }^{3}$ Department of Physics and Astronomy, University of Iowa, Iowa City, IA 52245, USA
}

Received: 24 September 2002 - Revised: 15 April 2003 - Accepted: 17 April 2003 - Published: 1 January 2004

\begin{abstract}
A study of Pc-5 magnetic pulsations using data from the Combined Release and Radiation Effects Satellite (CRRES) was carried out. Three-component dynamic magnetic field spectrograms have been used to survey ULF pulsation activity for the approximate fourteen month lifetime of CRRES. Two-hour panels of dynamic spectra were examined to find events which fall into two basic categories: 1) toroidal modes (fundamental and harmonic resonances) and 2) poloidal modes, which include compressional oscillations. The occurence rates were determined as a function of $L$ value and local time. The main result is a comparable probability of occurence of toroidal mode oscillations on the dawn and dusk sides of the magnetosphere inside geosynchronous orbit, while poloidal mode oscillations occur predominantly along the dusk side, consistent with high azimuthal mode number excitation by ring current ions.

Pc-5 pulsations following Storm Sudden Commencements (SSCs) were examined separately. The spatial distribution of modes for the SSC events was consistent with the statistical study for the lifetime of CRRES. The toroidal fundamental (and harmonic) resonances are the dominant mode seen on the dawn-side of the magnetosphere following SSCs. Power is mixed in all three components. In the 21 dusk side SSC events there were only a few examples of purely compressional (two) or radial (one) power in the CRRES study, a few more examples of purely toroidal modes (six), with all three components predominant in about half (ten) of the events.
\end{abstract}

Key words. Magnetospheric physics (MHD waves and instabilities; magnetospheric configuration and dynamics) Space plasma physics (waves and instabilities)

\section{Introduction}

Ultra-low-frequency (ULF) waves were first observed in ground-based magnetometer measurements more than a

Correspondence to: M. K. Hudson

(Mary.Hudson@dartmouth.edu) century ago by Stewart (1861) including the appearance of quasi-sinusoidal magnetic oscillations with periods of a few minutes (150-600 s, $2-7 \mathrm{mHz}$ frequency), now categorized as Pc-5 oscillations (Jacobs, 1964). In situ measurements of ULF oscillations, primarily at geosynchronous orbit, were reported in the 1960s. Statistical studies were performed with the ISEE 1 and 2 (Zhu and Kivelson, 1991), AMPTE CCE (Anderson et al., 1990) and AMPTE IRM (Lessard et al., 1999) satellites with more eccentric orbits. A number of studies using different devices such as ground magnetometers, radars, and geosynchronous satellites, have been carried out (see review by Hughes, 1994) prior to the ISEE and AMPTE studies. Since magnetometers measure the magnetic field at a single point, the definition of wave propagation direction and wavelength is ambiguous unless multipoint measurements are made (Takahashi et al., 1985) or wave electric field measurements are incorporated (Clemmons et al., 2000). Radar measurements have provided additional constraints on these parameters, radial localization and local time distribution (Walker et al., 1979; Fenrich et al., 1995). The purpose of these and the present CRRES study has been to understand the global morphology and to identify the generation mechanisms of ULF waves.

There are several properties of pulsations to be taken into account in such studies: frequency characteristics, including harmonic structure; spatial distribution; polarization properties; correlation with solar wind parameters; relation to geomagnetic activity, for example, phases of storms and substorms; correlation with particle signatures. ULF waves have a range of frequencies from $1 \mathrm{mHz}$ corresponding to the lowest frequency that the magnetospheric cavity can support to the ion gyrofrequency, which is in the $1 \mathrm{~Hz}$ range outside the plasmapause. The amplitude of the perturbations varies from fractions of a nanotesla to several hundred, with the largest amplitude usually occurring in the longer-period waves at high latitudes (auroral zone). A variety of mechanisms can produce ULF waves. Dungey (1954) first recognized that magnetic pulsations observed on the ground are hydromagnetic eigenmodes of approximately dipolar geo- 


\section{TORODIAL} MODE
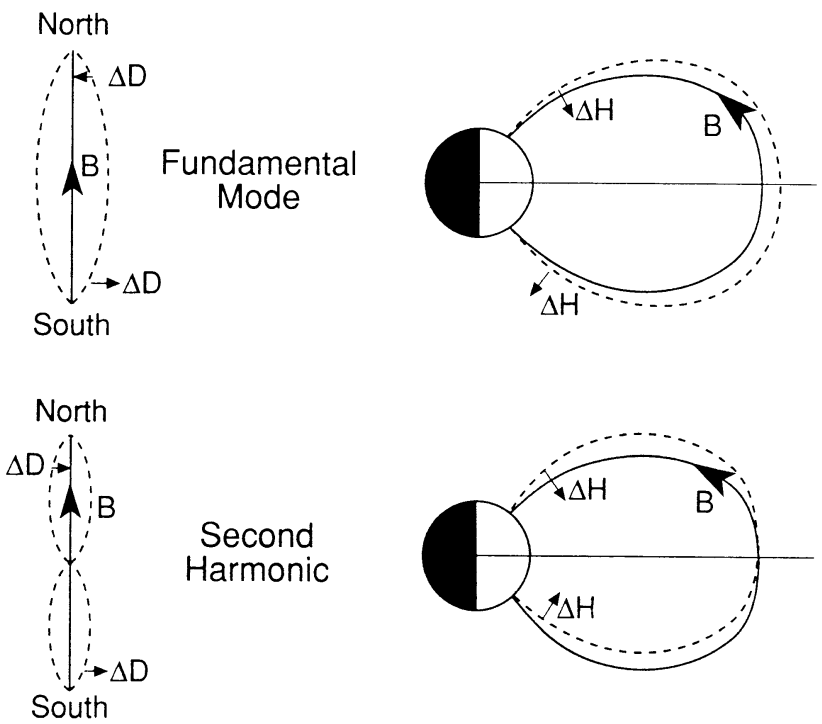

Fig. 1. Toroidal and poloidal mode fundamental and second harmonic perturbations sketched in a meridional plane, right, and looking earthward at a field line stretched from north to south, left (Hughes, 1994). $\Delta \mathrm{D}$ and $\Delta \mathrm{H}$ refer to east-west and north-south perturbations, respectively.

magnetic field lines. The physical model is that of field lines fixed at both ends in a perfectly conducting ionosphere, with harmonic standing waves between hemispheres whose frequency is a function of the length of the field line and number of nodes along the field line, as well as the plasma density and magnetic field which determine the Alfvén speed. Assuming that a wave propagates in the form of $\exp (i m \phi-i \omega t)$ in an axially symmetric field, where $\phi$ is the azimuthal phase angle and $m$ is the azimuthal mode number, we can write the full hydrodynamic wave equations in cylindrical coordinates in terms of the usual fluid density $\rho$, velocity u, electric and magnetic field variables as follows (Radoskii, 1967; Hughes, 1994):

$\omega^{2} \mu_{0} \rho-\frac{1}{r}(\mathbf{B} \cdot \nabla) r^{2}(\mathbf{B} \cdot \nabla)\left(\frac{u_{\phi}}{r}\right)=\omega m\left(\frac{\mathbf{B} \cdot \mathbf{b}}{r}\right)$

$$
\begin{gathered}
\left(\omega^{2} \mu_{0} \rho-r B^{2}(\mathbf{B} \cdot \nabla) \frac{1}{r^{2} B^{2}}(\mathbf{B} \cdot \nabla)\right)\left(r E_{\phi}\right)= \\
1 \omega B^{2}(\mathbf{B} \cdot \nabla)_{\phi} \frac{\mathbf{B} \cdot \mathbf{b}}{B^{2}}
\end{gathered}
$$

$i \omega \mathbf{B} \cdot \mathbf{b}=\frac{1}{r}(\mathbf{B} \cdot \nabla)_{\phi}\left(r E_{\phi}\right)-i m B^{2} \frac{u_{\phi}}{r}$.

The left-hand sides of the first two equations have derivatives along the magnetic field direction $\mathbf{b}, \mathbf{B} \cdot \nabla$ (defined in terms of the total magnetic field, background plus perturbed); they can be considered as one-dimensional wave equations.
The right-hand sides of both equations depend on the compressional part of the hydromagnetic wave. The third equation closes the set. Though the equations are coupled and have not been solved in general, with certain assumptions they can be solved for toroidal, poloidal and compressional mode waves.

In the limit that the azimuthal mode number $m$ goes to infinity, the product $\mathbf{B} \cdot \mathbf{b}$ must be zero, in order for the left-hand side of the first equation to be finite. Then, the right-hand side of the second equation vanishes and the equation describes a poloidal mode standing Alfvén wave (in a homogeneous system, the dispersion relation would be $\omega^{2}=k_{\|}^{2} V_{A}^{2}$, where $\left.V_{A}^{2}=B^{2} /\left(\mu_{0} \rho\right)\right)$. The corresponding fundamental and next (here labeled second) harmonic poloidal modes are shown on the right side of Fig. 1.

The electric field varies azimuthally (east-west) and the magnetic field oscillates radially. For this limit, perturbations on a meridional plane can be considered to be decoupled from those on adjacent planes.

In the $m=0$ limit, the first two equations decouple and two independent solutions result. The first equation describes the toroidal mode. The left side of Fig. 1 illustrates the two lowest harmonics of this mode. The magnetic field and velocity oscillate in the azimuthal direction, while the electric field has radial fluctuations. Each L-shell oscillates azimuthally independent of the others. The second equation describes the compressive (or fast) mode.

If $m$ goes to 0 but is not equal to it, modes described by Eq. (1) and (2) can couple. Physically, field lines with standing Alfvén wave frequencies matching one of the cavity mode eigenfrequencies may be excited (Goldstein et al., 1999). Thus, the energy of the magnetospheric cavity transfers to field line resonances. Ultimately, ULF pulsations derive their energy from the solar wind. Pulsations produced either in the solar wind/magnetosheath or at the magnetopause/boundary layer (so-called "upstream source") may couple energy directly from the solar wind, while the ring current can act as an intermediary in transferring solar wind energy. It is important to determine whether the source of the waves is external or internal to the magnetosphere (Fenrich et al., 1995), in order to understand the efficiency and timescale of solar wind driven excitation of ULF waves. Those modes, typically low $m$ number toroidal oscillations, excited directly by upstream solar wind perturbations and velocity shear instability at the magnetopause (Miura, 1992), will respond more immediately to changing solar wind conditions than internal instabilities requiring a buildup of the ring current. Internal instabilities of high $m$-number poloidal modes are excited by ring current ions injected into the dusk to noon sector (Southwood, 1976; Chen and Hasegawa, 1991), which, in turn have been energized by the convection electric field imposed by the solar wind. According to their origin, Pc perturbations have been classified as in Table 1 (Anderson, 1994).

The focus of the present study is on Pc-5 oscillations, particularly poloidal and toroidal Pc-5 modes. Poloidal Pc-5 
Table 1. Category, location and source of different types of Pc 3-5 oscillations (based on Anderson, 1994).

\begin{tabular}{llll}
\hline Category & Location & Source & Note \\
\hline Compressional Pc3 & Day-side & Upstream & \\
Toroidal Pc3 & Day-side & Upstream & \\
$\begin{array}{l}\text { or multi harmonics } \\
\text { Poloidal Pc4 }\end{array}$ & Afternoon evening & Local & \\
$\begin{array}{l}\text { Compressional Pc-5 } \\
\text { (Poloidal) }\end{array}$ & $\begin{array}{l}\text { Night-side, } \\
\text { dawn and dusk }\end{array}$ & Local & $\begin{array}{l}\text { Related to } \\
\text { ion injections }\end{array}$ \\
Toroidal Pc-5 & $\begin{array}{l}\text { Dawn and dusk } \\
\text { flanks }\end{array}$ & Upstream & $\begin{array}{l}\text { Fundamental } \\
\text { mode field line } \\
\text { resonances }\end{array}$ \\
\hline
\end{tabular}

waves with power in both radial and compressional components were first observed in situ and reported by Sonnerup et al. (1969), based on Explorer 26 magnetometer data. These waves commonly occur on the dusk-side of the magnetosphere. It has been shown that poloidal Pc5 pulsations may be generated by the pressure anisotropy of the ions, which drives local wave-particle instability (Southwood, 1974; Chen and Hasegawa, 1974). The excitation mechanism is fundamentally kinetic for a plasma beta (ratio of kinetic to magnetic pressure) typical of the ring current, involving drift bounce resonance of ring current ions with high ( $\mathrm{m} \sim 100)$ azimuthal mode number oscillations.

Toroidal mode Pc-5 waves are low $m$ number field line resonances (see review by Glassmeier, 1995). Statistical studies using radar and ground magnetometers have shown that toroidal mode Pc-5s have a peak occurrence on the dawn-side of the magnetosphere. Some satellite studies have shown greater dawn-dusk symmetry (Glassmeir and Stellmacher, 2000), while others support a higher probability of occurrence on the dawn-side (Anderson et al., 1990; Lessard et al., 1999; Takahashi et al., 2002). Toroidal mode Pc-5 waves have been attributed to excitation by the Kelvin-Helmholz instability due to velocity shear, which is greatest along the flanks of the magnetosphere (Miura, 1992).

\section{Observations}

The Combined Release and Radiation Effects Satellite (CRRES) was launched on 25 July 1990, at 19:21 UT into a geosynchronous transfer orbit, with an apogee near geosynchronous orbit (actually $6.3 R_{E}$, but larger in L), perigee at $350 \mathrm{~km}$, an orbital period of $9.9 \mathrm{~h}$ and an inclination of 18.2 degrees relative to the equatorial plane. The spin axis was oriented within 15 degrees of the Sun such that the solar panels (located on top of the spacecraft) were continuously illuminated by the Sun and the spin period was about $30 \mathrm{~s}$. The orientation and relatively low spin rate provided very low levels of solar-array interference as compared to most other magnetospheric spacecraft. In the approximate fourteen months that CRRES operated, the magnetic local time (MLT) of apogee regressed at a rate of $15 \mathrm{~h} / \mathrm{yr}$ from $\approx 08: 00$ MLT through midnight to 13:30 MLT, so a complete local time sweep was not covered. CRRES carried a wide range of particle detectors and a triaxial fluxgate magnetometer mounted at the end of a $6.1 \mathrm{~m}$ boom (Singer et al., 1992), and a plasma wave receiver connected to a $100 \mathrm{~m}$ long-wire electric dipole antenna perpendicular to the spin axis (Anderson et al., 1992). The magnetic field data was sampled at a rate of 16 samples per second. The telemetered data stream in sensor coordinates was routinely calibrated and transformed into a 2-s data base in a VDH coordinate system. In this cylindrical system, $\mathrm{H}$ is anti-parallel to the magnetic dipole axis (parallel to the magnetic field at the equator), $\mathrm{V}$ is radially outward in the magnetic equatorial plane (in the magnetic meridian perpendicular to $\mathrm{H}$ ), and D is eastward, completing the set. The spin axis of CRRES was roughly parallel to the x-component of the GSE system. The magnetometer was capable of operating in two resolution modes and was controlled automatically, however, for the data used in this analysis, it was operated in high gain mode with a sensitivity of $0.43 \mathrm{nT}$ (for fields $<850 \mathrm{nT}$ ).

The total observation time is presented in Fig. 2, binned in equatorial plane radial distance (referred to here as L-shell) and local time. The hatched bins are those where CRRES spent less than $9 \mathrm{~h}$. As can be seen, while the CRRES satellite covered most local times and L-shells in a range from 2 to 9 , it spent more time on the dawn and dusk sides of the magnetosphere and nightside, with little coverage on the dayside around noon.

The magnetic field data with a 2 -s resolution was first linearly interpolated to fill the gaps of $120 \mathrm{~s}$ or less and then averaged over a period of $30 \mathrm{~min}$, to obtain the background magnetic field. For each data point, a segment of 900 data entries centered on a given data point was fitted with a straight line. The value of the midpoint of the fitted line was taken to be the magnitude of the background magnetic field at the time of the given data point. The segment of 900 data points was chosen in order to remove the background field without reducing Pc-5 pulsation amplitudes. The calculated 


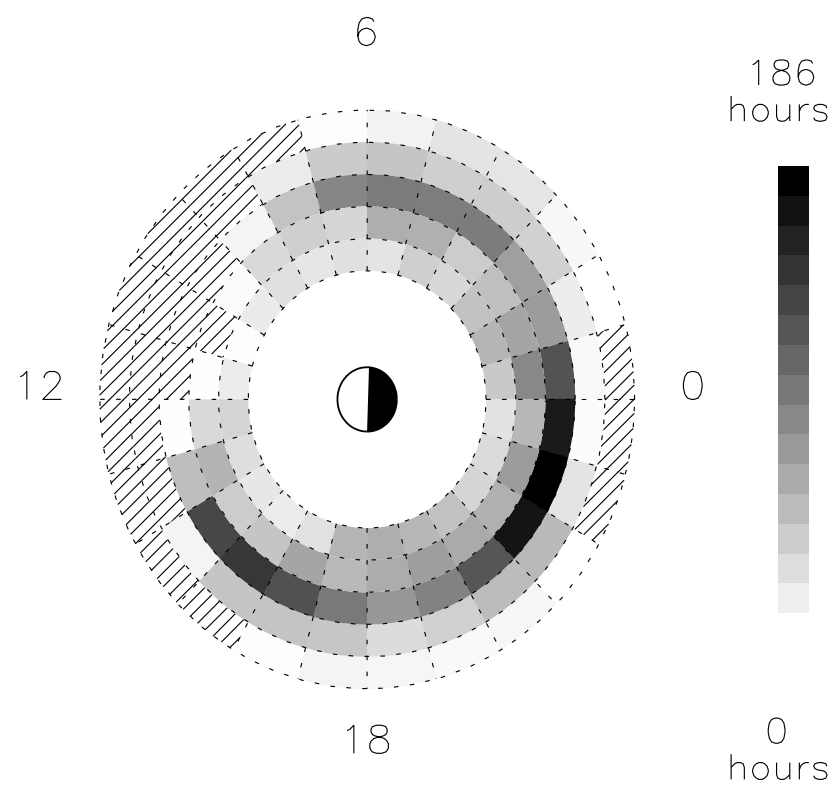

Fig. 2. Total observation time spent in each L-LT bin $(L=4-9)$ by the CRRES satellite.

background field was subtracted from the original data and the obtained detrended data were used to calculate the dynamic spectra. For each component, segments of 256 points were extracted and a Hanning window was applied (Lessard et al., 1999). The spectral power was calculated using a fast Fourier transform. The calculations were repeated until enough spectra were accumulated to fill two-hour panels. The power spectral density was then plotted using a log scale that spans several orders of magnitude to cover the range in wave power. The magnetic local time, L-shell and magnetic latitude are included on the plot. The plots of detrended magnetic field data and its dynamic spectra were visually scanned to find various events. The data were compared with the fundamental field line resonance (FLR) frequency calculated using the electron density determined from measurements by the plasma wave instrument on CRRES (Anderson et al., 1992) of either the upper hybrid resonance or the electron plasma frequency. Physically, the FLR model is a standing Alfvén wave with endpoints tied to an assumed perfectly conducting ionosphere. Figure 1 shows examples of harmonics.

Calculation of the normalized FLR toroidal mode fundamental frequency from the measured plasma density and background magnetic field is described by Schulz (1996) or Denton and Gallagher (2000), using the mass factor (ratio of mass density to electron density) from the Global Core Plasma Model (GCPM) (Gallagher et al., 2000). (In the GCPM, the mass factor is close to 1.15 in the regions we will be considering.) This frequency is superimposed on the spectral plots of each field component shown later to assist in mode identification. In the following section we present a statistical analysis of Pc-5 oscillations as measured by the CRRES magnetometer and sorted by toroidal and poloidal polarization in the VDH coordinate system. An analysis of Storm Sudden Commencement (SSC) events during the 14-month lifetime of CRRES is then summarized. In the absence of continuous solar wind measurements during the lifetime of CRRES, SSCs were chosen to enable a focus on sudden changes in solar wind conditions as a driver of ULF wave oscillations. This was a particularly active time period around the Cycle 22 solar maximum, rich in SSC events.

\section{Statistical study}

The purpose of this study is to determine the occurrence rates of different types of pulsations over all local times in the region from $\mathrm{L}=2$ to $\mathrm{L}=10$ for the lifetime of CRRES (14 months). Two types of events were considered in the study: toroidal and poloidal mode pulsations, including both fundamental and harmonic resonances. Plots with dynamic spectra and magnetic field data along with coordinate plots were visually examined to find those events. The duration of the events, the magnetic local time and the position of the satellite were recorded. The resulting occurrence rates were normalized to the total observation time for each space bin (L-shell, MLT), shown in Fig. 2.

\section{Toroidal pulsations}

Toroidal pulsations are the most frequently observed and well-defined magnetic pulsations. In this study the definition given by Anderson et al. (1990) was used to identify fundamental and harmonic resonances. The narrow band signal, with $\Delta f<2 \mathrm{mHz}$ seen in the toroidal component and frequency decreasing as L-shell increases (Samson and Rostoker, 1972), is considered to be the resonance. If the frequency is less than $10 \mathrm{mHz}$, the event is identified as a fundamental resonance, otherwise as a harmonic. An example of such an event is shown in Fig. 3 for the time interval 08:0010:00 UT, orbit 81, 28 August 1990. The first two panels show the dynamic spectra and detrended magnetic field data radial component ( $V$ component). The second set presents the eastward component (D) and the third is the compressional (parallel to B) component (H). The next three plots show the magnetic local time (MLT), L-shell and magnetic latitude (Mlat). The black curve on the dynamic spectra represents the fundamental Field Line Resonance (FLR) frequency calculated using local density determined from the upper hybrid resonance or electron plasma frequency measured by the plasma wave instrument on CRRES (Anderson et al., 1992). The frequency range on the dynamic spectra is $0-20 \mathrm{mHz}$, chosen in order to see the signature of Pc-5 oscillations. The magnetic field range is $-20 \mathrm{nT}$ to $20 \mathrm{nT}$ in this example, $-5 \mathrm{nT}$ to $5 \mathrm{nT}$ or $-50 \mathrm{nT}$ to $50 \mathrm{nT}$ in other examples, depending on the maximum value of the magnetic field perturbation for a given interval. A well-defined signal is seen in the eastward magnetic field component for the time interval 08:30-10:00 UT. The frequency of the pulsation is about $3 \mathrm{mHz}$, with a $2 \mathrm{mHz}$ bandwidth, and is consistent 


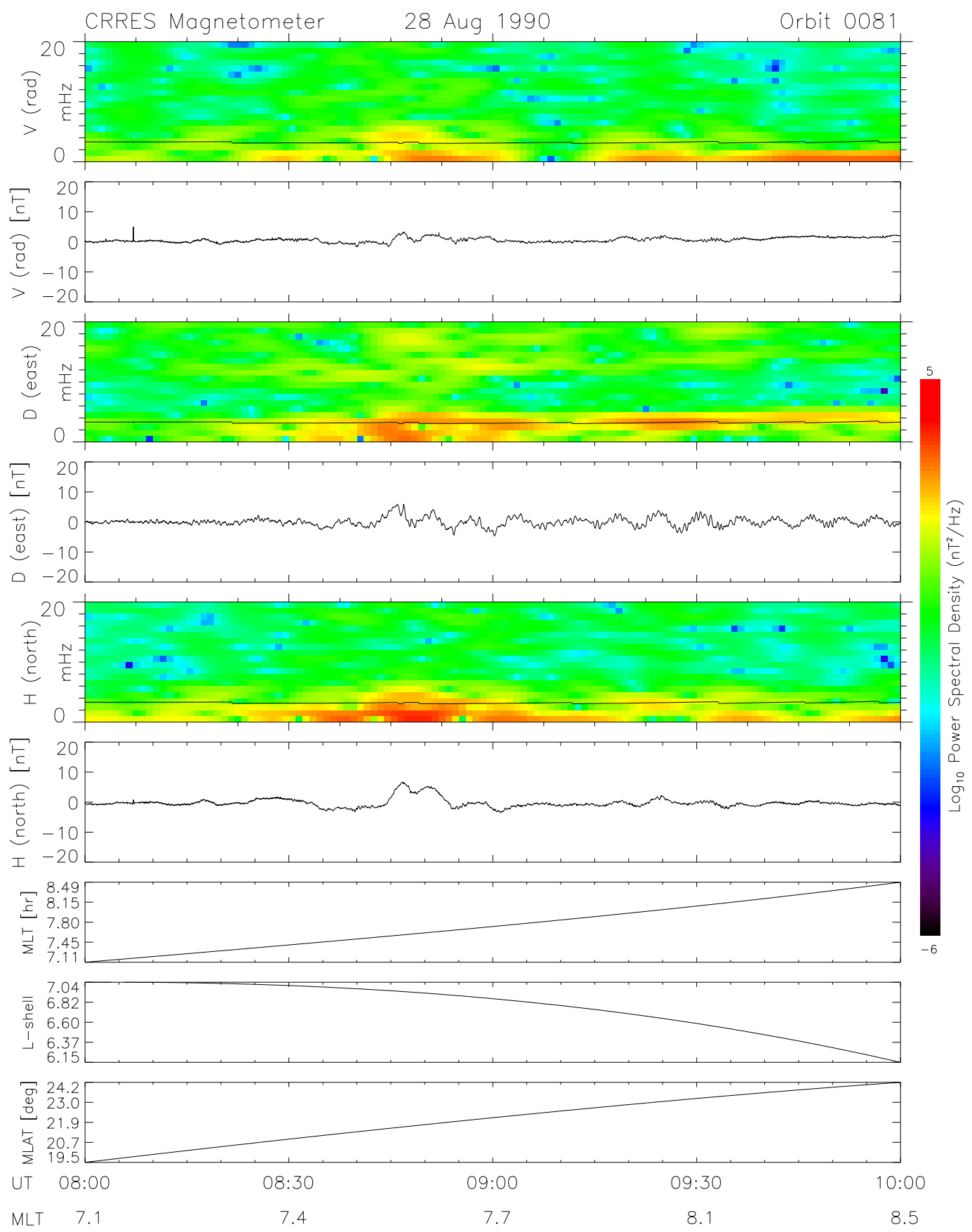

Fig. 3. Sample of toroidal pulsation measured by the CRRES magnetometer on 28 August 1990. See Toroidal Pulsations text for detailed description of panels. 


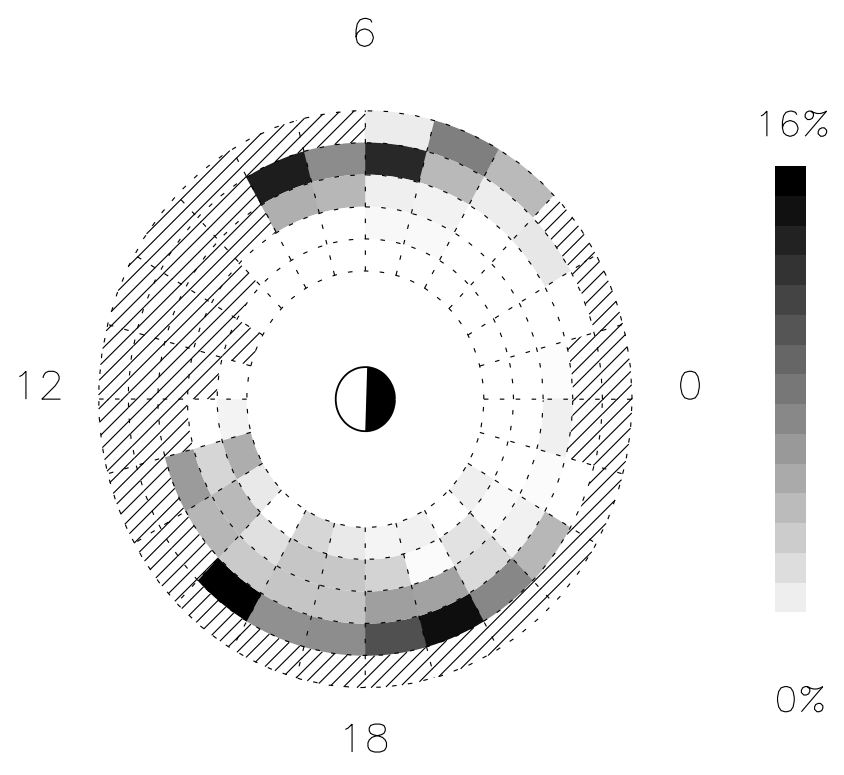

Fig. 4. Occurence rates of toroidal Pc-5 pulsations measured by CRRES.

with the theoretical fundamental FLR frequency, indicated by the solid curve. The amplitude is $5 \mathrm{nT}$, which is common for satellite measurements of toroidal Pc-5 oscillations near geosynchronous orbit. The $\mathrm{L}$ dependence of the fundamental frequency can be observed. While the L-shell is decreasing from 7 to 6.15 , the fundamental frequency slowly increases from $3 \mathrm{mHz}$ to $4 \mathrm{mHz}$. Along with the fundamental resonance, the third and fourth harmonics are seen in the toroidal component with frequencies of $12 \mathrm{mHz}$ and $17 \mathrm{mHz}$, respectively. The second harmonic which has an equatorial maximum could not be observed at CRRES because the satellite had an 18.2 degree inclination. (These identifications are consistent with a mass density distribution $\rho=\rho_{0}\left(L R_{E} / R\right)^{1}$, where $\rho_{0}$ is the equatorial mass density (Schulz, 1996; Denton and Gallagher, 2000).) These harmonics are observed for the time periods 08:55-09:10 UT and 09:28-09:38 UT. No signal is seen in the poloidal components consistent with the fundamental or harmonics.

The statistical distribution of the fundamental toroidal resonance is presented in Fig. 4, normalized by the observation time spent in each L-LT bin (Fig. 2). As seen, toroidal oscillations tend to occur on the dawn and dusk sides of the magnetosphere at $\mathrm{L}=6$ to 8 for the CRRES study. Lack of spatial coverage between 08:00-13:00 LT (Fig. 2) precludes a conclusion about dayside occurence, but there is a clear absence of fundamental toroidal mode power near midnight. The local time distribution is consistent with the comparable dawn-dusk occurence of narrow band Pc-5 wave power reported by Glassmeier and Stellmacher (2000) based on geosynchronous GOES 6 data, while the AMPTE IRM study by Lessard et al., (1999) found more events on the dawn than dusk side of the magnetosphere at higher L-values, as did the AMPTE CCE study of Anderson et al., (1990) and Takahashi et al., (2002). The apogee of CRRES was at $6.3 R_{E}$, so the satellite spent most of its time at L-values between 5 and 8 , while AMPTE IRM had an apogee at $18 R_{E}$, and thus crossed low L-shells rapidly. AMPTE CCE, with a $9 R_{E}$ apogee, was intermediate in L-coverage. AMPTE IRM also had a higher geographic inclination (28.6 degrees) than CRRES (18.2 degrees), while AMPTE CCE was lower (4.8 degrees), which can affect the probability of observing modes with an equatorial or near-equatorial node (Denton et al., 2001).

The interpretation of toroidal mode FLRs as standing Alfvén waves is well suported by our calculation of the fundamental frequency based on the CRRES plasma wave instrument determination of local plasma density. The dawndusk occurrence has been attributed to the excitation of surface waves on the magnetopause by the Kelvin-Helmholtz instability (Miura, 1992). Coupling of surface wave energy into field line resonances has been examined by Chen and Hasegawa (1974). Figure 4 is consistent with other studies in finding a greater probability of occurrence at higher L-values, closer to the magnetopause source (Anderson et al., 1990; Lessard et al., 1999). Ground-based observations of Pc-5 pulsations show a strong dawn-dusk asymmetry which has been attributed to, among other things, ionospheric screening of shorter wavelength modes on the dusk side (Yumoto et al., 1983), local time dependence of field line resonance widths (Glassmeier and Stellmacher, 2000) and a variation in the instability at the magnetopause. The critical velocity threshold for the Kelvin-Helmholz instability increases with the intensity of the magnetosheath magnetic field (Lee et al., 1981), which is greater at the dusk side due to magnetosheath compression. Since the magnetosheath flow velocity increases along the flanks of the magnetosphere from an ideal subsolar stagnation point, the flow may be unstable farther downstream on the dusk side than dawn-side, due to magnetosheath magnetic field compression (Lee et al., 1981), leading to the type of asymmetry observed by Lessard et al. (1999, Fig. 6).

\section{Poloidal pulsations}

Poloidal mode pulsations are characterized by a narrow band signal in the radial and compressional components with frequencies less than $10 \mathrm{mHz}$. Figure 5 shows an example of this type of event. The pulsation starts at 22:15 UT and lasts for half an hour. It has an amplitude of $9 \mathrm{nT}$ and a frequency of $4-5 \mathrm{mHz}$. The signal is $3 \mathrm{mHz}$ wide and is about equal to the theoretical fundamental FLR frequency (black curve). For zero pressure, poloidal pulsations are expected to have slightly lower FLR frequencies than the toroidal mode, as discussed in Cummings et al. (1969). A larger effect (for odd modes, not necessarily the fundamental) occurs when an inward pressure gradient reduces the poloidal but not the toroidal mode frequency (Vetoulis and Chen, 1996). The agreement here of the observed poloidal mode frequency and that of the fundamental toroidal mode argues that the observed wave may have fundamental structure along the field 


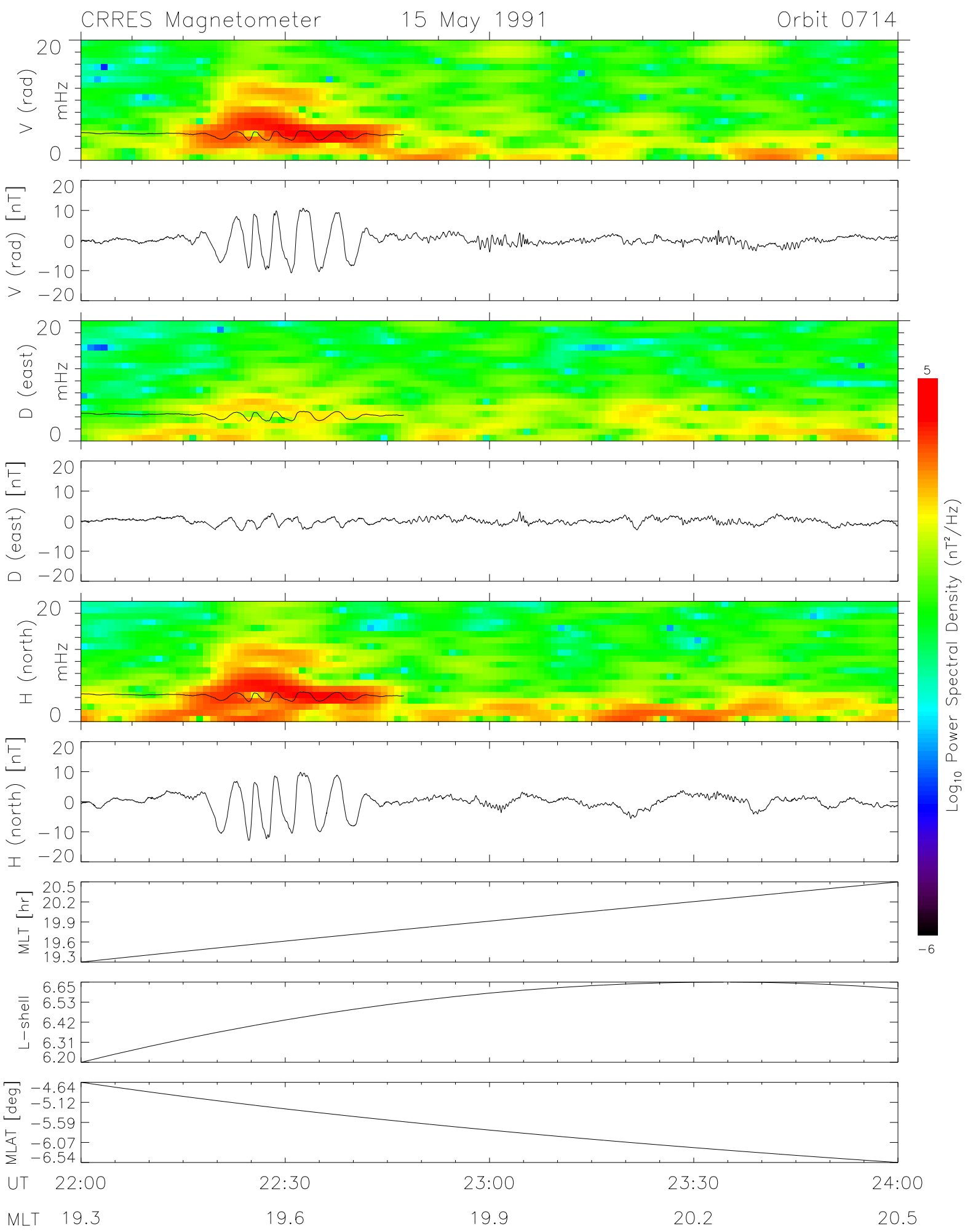

Fig. 5. Sample of poloidal Pc-5 pulsations measured by the CRRES magnetometer on 15 May 1991, same format as Fig. 3. 


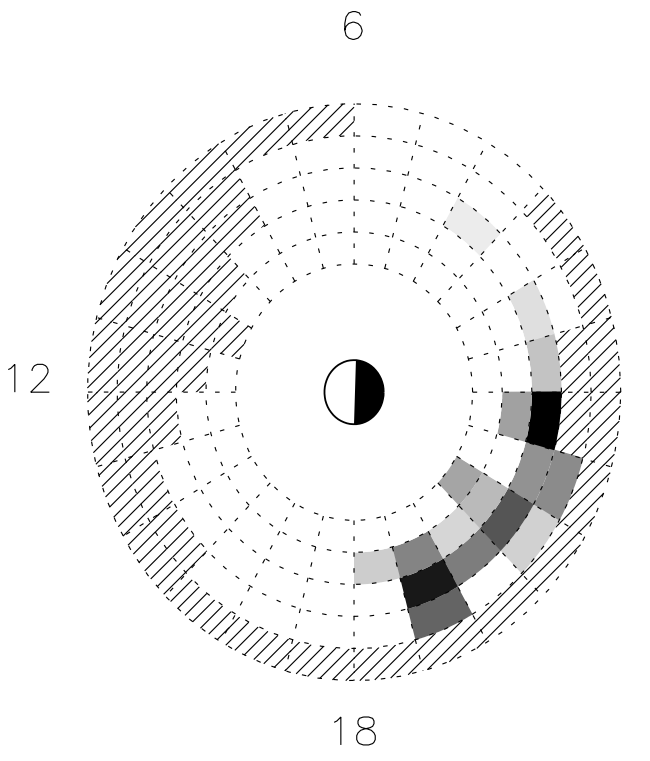

$0 \%$

Fig. 6. Occurence rates of poloidal Pc-5 pulsations measured by CRRES.

line, although on theoretical grounds, we would expect the second harmonic (Cheng and Lin, 1987). The black curve in Fig. 5 shows a variation in frequency which most likely results from the large amplitude oscillation in $B$. This observation is rare and not a necessary feature of poloidal pulsations. While there is strong evidence of oscillations in radial and compressional components with the same shape, the dynamic spectra in the eastward (toroidal) component shows very weak activity. Other events similar to the one shown in Fig. 5 were found and recorded.

Figure 6 shows the occurrence rates of poloidal pulsations. The oscillations occur predominantly on the dusk side of the magnetosphere. None are seen at noon, where spacecraft coverage was limited (Fig. 2). Higher occurence rates are evident at $\mathrm{L}=7-9$. This distribution suggests ion injection as a possible source of poloidal mode oscillation. Ions injected into the ring current during geomagnetic storms produce high $m$ number poloidal modes, as they drift around the dusk side, by a drift-bounce resonant kinetic interaction (Southwood et al., 1969; Chen and Hasegawa, 1991). Dipolarization during substorms and bursty bulk flows will also produce a poloidal mode signature in the evening sector (Lessard et al., 1999).

\section{Storm Sudden Commencement (SSC) events}

In this section several Storm Sudden Commencement (SSC) events are discussed. Times were chosen according to the A-Quality ("very remarkable") SSC list from the IAGA Bulletin, Geomagnetic Data 1990-91 (Table 2).

Classification of the SSC events was based on low latitude ground magnetometer observatories. An SSC or sudden impulse (SI) is defined as a sudden increase in the geomagneticfield intensity of a few tens or more nanotesla, caused ultimately by an increase in solar-wind dynamic pressure. This
Table 2. SSC List.

\begin{tabular}{rrrr}
\hline Date & UT & Date & UT \\
\hline 01 Aug. 1990 & $07: 41$ & 04 Jun. 1991 & $03: 37$ \\
26 Aug. 1990 & $05: 43$ & 07 Jun. 1991 & $22: 28$ \\
29 Aug. 1990 & $11: 22$ & 09 Jun. 1991 & $00: 40$ \\
01 Sep. 1990 & $12: 39$ & 10 Jun. 1991 & $17: 16$ \\
26 Oct. 1990 & $11: 34$ & 12 Jun. 1991 & $10: 12$ \\
29 Oct. 1990 & $20: 11$ & 17 Jun. 1990 & $10: 19$ \\
26 Nov. 1990 & $23: 32$ & 17 Jun. 1991 & $19: 22$ \\
08 Dec. 1990 & $14: 25$ & 30 Jun. 1991 & $01: 16$ \\
12 Jan. 1991 & $01: 51$ & 06 Jul. 1991 & $15: 28$ \\
01 Feb. 1991 & $18: 42$ & 08 Jul. 1991 & $16: 36$ \\
04 Feb. 1991 & $22: 14$ & 12 Jul. 1991 & $09: 24$ \\
04 Mar. 1991 & $16: 19$ & 19 Jul. 1991 & $19: 04$ \\
09 Mar. 1991 & $22: 45$ & 30 Jul. 1991 & $05: 57$ \\
21 Mar. 1991 & 06:00 & 02 Aug. 1991 & $05: 33$ \\
24 Mar. 1991 & $03: 41$ & 05 Aug. 1991 & $20: 46$ \\
04 Apr. 1991 & $11: 22$ & 11 Aug. 1991 & $02: 53$ \\
19 Apr. 1991 & $10: 56$ & 18 Aug. 1991 & $18: 33$ \\
24 Apr. 1991 & 20:45 & 20 Aug. 1991 & $08: 00$ \\
13 May 1991 & $08: 57$ & 27 Aug. 1991 & $15: 15$ \\
16 May 1991 & 20:41 & 01 Oct. 1991 & $18: 14$ \\
22 May 1991 & 00:18 & 07 Oct. 1991 & $13: 58$ \\
31 May 1991 & $10: 39$ & 17 Oct. 1991 & $13: 31$ \\
\hline
\end{tabular}

sudden increase is called an SSC if the event is followed by a storm and an SI, if it is not.

During the lifetime of CRRES, there were 44 such SSC events from which we have excluded 13 due to the absence of data, invalid measurements, for example, near perigee where the background magnetic field strength precludes an accurate measurement of the fluctuating field, or the presence of very strong activity which saturates the measurement of FLR mode structure. The spatial distribution of the remaining 31 events are shown in Fig. 7.

The events are color coded by their dominant polarization, with toroidal (red), compressional (green), radial (light blue), all three components (black) and no response (yellow). Open circles indicate less certainty in the identification of the modes, but a clear presence of ULF oscillations in the $1-10 \mathrm{mHz}$ range. The dawn-side SSC events were clearly dominated by toroidal mode oscillations (red), while there is a mixture of polarizations observed on the dusk side. The predominant noon to dusk signature is power in all three modes (black).

For each event we examined 2-s CRRES detrended magnetic field data, its dynamic spectra and orbital summary plots for the appropriate time interval. Two are presented as examples, 26 August 1990, because of the availability of simultaneous IMP 8 solar wind data, and 1 September 1990 , as an example of a double SSC interval.

\subsection{August 1990}

A sample SSC event occurred at 05:43 UT, orbit 76, 26 August 1990. Figure 8 shows data for the interval 


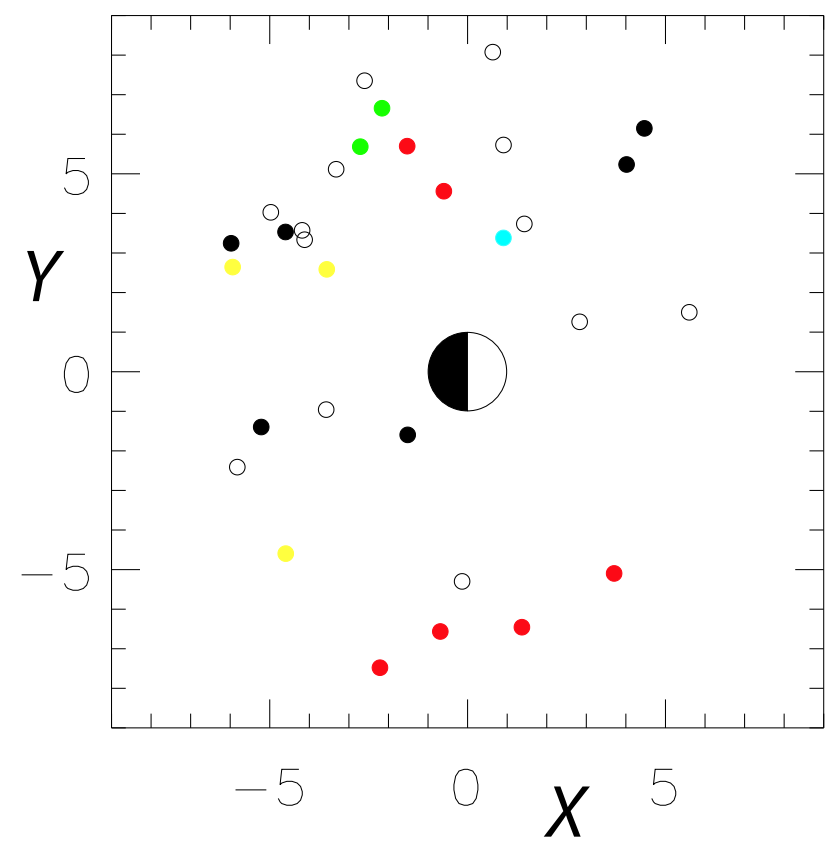

Fig. 7. CRRES SSC events color coded by their dominant polarization, with toroidal (red), compressional (green), radial (light blue), all three components (black) and no response (yellow). Open circles indicate less certainty in identification of the modes.

05:20-08:00 UT. The CRRES satellite was near apogee at $\mathrm{L}=6.3$, MLT $=6.8$ and Mlat $=12.0$ degrees at the time of the SSC. The magnetic field increased in 3 min by $14.5 \mathrm{nT}$ in the compressional component, $11 \mathrm{nT}$ in the radial and by $7 \mathrm{nT}$ in the eastward component, with a rise of $22 \mathrm{nT}$ in the total field. Oscillations are evident in all three components for almost $8 \mathrm{~h}$. The toroidal oscillation, with amplitude of $3 \mathrm{nT}$, is seen for the interval 05:32-05:43 UT. The dynamic spectra show power around $7 \mathrm{mHz}$, which is consistent with the theoretical fundamental FLR frequency calculated from the local plasma density measurement (solid curve). A precursor toroidal oscillation of amplitude 1-2 $\mathrm{nT}$ at the same fundamental frequency indicated by the line plot is seen prior to the SSC, greatly amplified and lasting about 90 minutes after the SSC, along with enhanced power in the poloidal (radial and compressional) components. IMP 8 data at $x=0, y=-30 R_{E}$, $z=5 R_{E}$ in GSM coordinates, along the dawn-side of the magnetosphere, is shown in Fig. 9.

These data show evidence of solar wind $B x$ oscillations prior to the SSC in the same frequency range as the precursor oscillations evident at CRRES in $B y$ (eastward component), both following a sudden increase in solar wind $B x$ at 05:26 UT. The data also shows a sudden increase in the solar wind speed and density that corresponds to the arrival of the interplanetary shock at 05:43 UT, responsible for the SSC.

In the toroidal component of Fig. 8 we can also see the presence of the second harmonic during the intervals 05:4006:00 UT and 06:10-06:40 UT. The frequency of this pulsation is about $16-18 \mathrm{mHz}$ for the first period and $15-17 \mathrm{mHz}$ for the second one. Presence of the harmonic is seen in the radial component for the first period with a frequency of $17 \mathrm{mHz}$. The theoretically calculated frequency for the second harmonic is $18.4 \mathrm{mHz}$ in this case.

\subsection{September 1990}

Figure 10 shows plots for 10:10-13:00 UT, orbit 91, 1 September 1990, with an example of a double SSC, a "BQuality" (fair, ordinary but unmistakable) SSC at 10:24 UT followed by an "A-Quality" SSC at 12:39 UT, from IAGA Bulletin, Geomagnetic Data 1990-91, Table 2). Such events are not uncommon, caused by forward and reverse solar wind shocks, resulting from a steepening of a disturbance propagating upstream and downstream in the solar wind frame, away from a discontinuity (Holzer, 1979). When the second SSC occurred at 12:39 UT, CRRES was near apogee at $\mathrm{L}=7.68$, Mlat $=28.2$ degrees and $\mathrm{MLT}=8.3$. Following the first SSC, toroidal oscillations are evident with a frequency fitting the theoretical fundamental FLR calculation (black curve). This pulsation starts at 10:24 UT and lasts for $2 \mathrm{~h}$, continuing after the second SSC. The amplitude after the first SSC is about $5 \mathrm{nT}$, and it increases to $20-50 \mathrm{nT}$ after the second, stronger SSC. The frequency of the pulsation slowly increases from $7 \mathrm{mHz}$ at $12: 00 \mathrm{UT}$ to $8 \mathrm{mHz}$ at $13: 05 \mathrm{UT}$. There are weak and long period (below the fundamental) oscillations in the toroidal and compressional components prior to the first SSC at 10:24 UT, but there is no solar wind data available for this period for comparison.

After the second SSC, power is evident in all three components during the time interval 12:35-13:00 UT. There is an average increase in the fundamental mode frequency due to increasing magnetic field strength, as indicated by the line plot on the color spectrograms, as CRRES moved radially inward in L. Any decrease in frequency due to increasing density as the plasmapause is approached is more subtle, as one might expect from the stronger dependence of Alfvén speed, which determines the standing wave frequencies, on $B$ rather than on $\sqrt{\rho}$.

From the 31 SSC events analyzed, 10 were observed when CRRES was post midnight, on the dawn-side of the magnetosphere; 21 were observed when CRRES was premidnight on the dusk side, reflecting the large number of SSC events from March - October, 1991, and rotation of the CRRES orbital plane. A comparison of Fig. 7 with Fig. 2 shows that CRRES sampled SSC at all local times covered by the orbit. Figure 7 shows that dawn-side SSCs excite predominantly toroidal modes (red), and there is mixture of polarizations observed on the dusk side. The predominant noon to dusk signature is power in all three modes (black).

\section{Discussion and conclusions}

Analysis of magnetometer data from the CRRES satellite has been presented in two parts: a statistical study of the polarization and spatial distribution of modes in $\mathrm{L}$ and local time, independent of geomagnetic conditions; and a study of time 


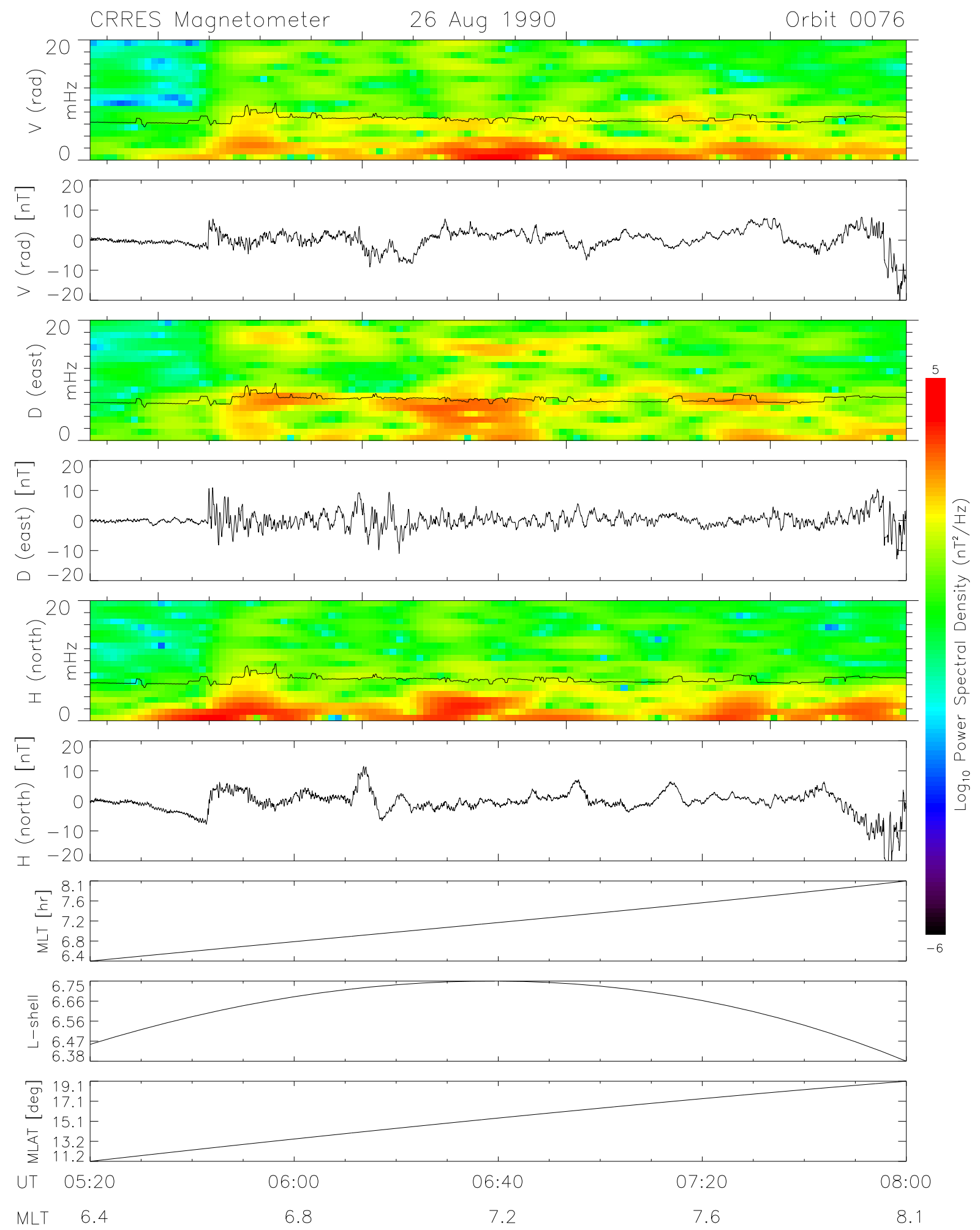

Fig. 8. SSC event of 26 August 1990, same format as Fig. 3. 
intervals marked by the occurence of Storm Sudden Commencements.

From the statistical survey for all conditions we conclude that:

1. Toroidal magnetic pulsations are fundamental field line resonances that match the frequency calculated from a determination of the local plasma density based on the upper hybrid resonance frequency or the electron plasma frequency measured by the CRRES plasma wave instrument (Anderson, 1992). While there have been many studies, going back to Cummings et al. (1969), which compare measured Pc-5 ULF wave frequencies with model calculations, few have used local plasma density measurements to validate the calculation (Denton et al., 2001).

2. Toroidal mode power is distributed fairly equally between the dawn and dusk flanks of the magnetosphere inside $\mathrm{L}=8$, with greater probability of occurrence at higher L-values. The former result differs from AMPTE CCE and AMPTE IRM satellite studies at higher L-values at solar minimum (Anderson et al., 1990; Lessard et al., 1999; Takahashi et al., 2002), but is consistent with a recent analysis of a six month interval of GOES 6 geosynchronous data in 1993, a period characterized by 27-day recurring high speed solar wind streams characteristic of the declining phase of the solar cycle (Knipp et al., 1998). The occurence of Pc-5 oscillations has been correlated with high speed solar wind periods (Engebretson et al., 1998; O'Brien et al., 2001), providing some support for the Kelvin-Helmholtz theory of toroidal mode FLR excitation. The high occurrence rate of toroidal modes on the dusk side in the CRRES study may be affected by the large occurrence rate of SSCs during the March - October, 1991 phase of the CRRES orbit, see Table 2. To examine this posibility, a separate study of the SSC polarizations was undertaken.

3. Poloidal modes were observed to occur premidnight, consistent with the distribution of storm-time pulsations (Anderson et al., 1990; Lessard et al., 1999) and the injection of ring current ions as a source population for exciting high $\mathrm{m}$ number poloidal modes via drift bounce resonance (Southwood et al., 1969; Chen and Hasegawa, 1988). They could also be signatures of substorm dipolarization or bursty bulk flows (Lessard et al., 1999).

The study of SSC events provides two main conclusions:

1. The toroidal fundamental (and harmonic) resonances are the dominant mode seen on the dawn-side of the magnetosphere following a Storm Sudden Commencement (SSCs).

2. Power is mixed in all three components on the dusk side following SSCs, with few examples of purely compressional (two) or radial (one) power in the CRRES study, and more examples of purely toroidal (six) modes, but all three components predominant (ten) in the 21 dusk-side SSCs. The magnetosphere is more strongly disturbed during SSC periods, with the injection of ring current ions available to drive poloidal modes in the dusk sector superimposed on any magnetopause boundary-driven toroidal oscillations. It is not surprising that the SSC polarization characteristics indicated in Fig. 7 combine the spatial distribution of modes integrated

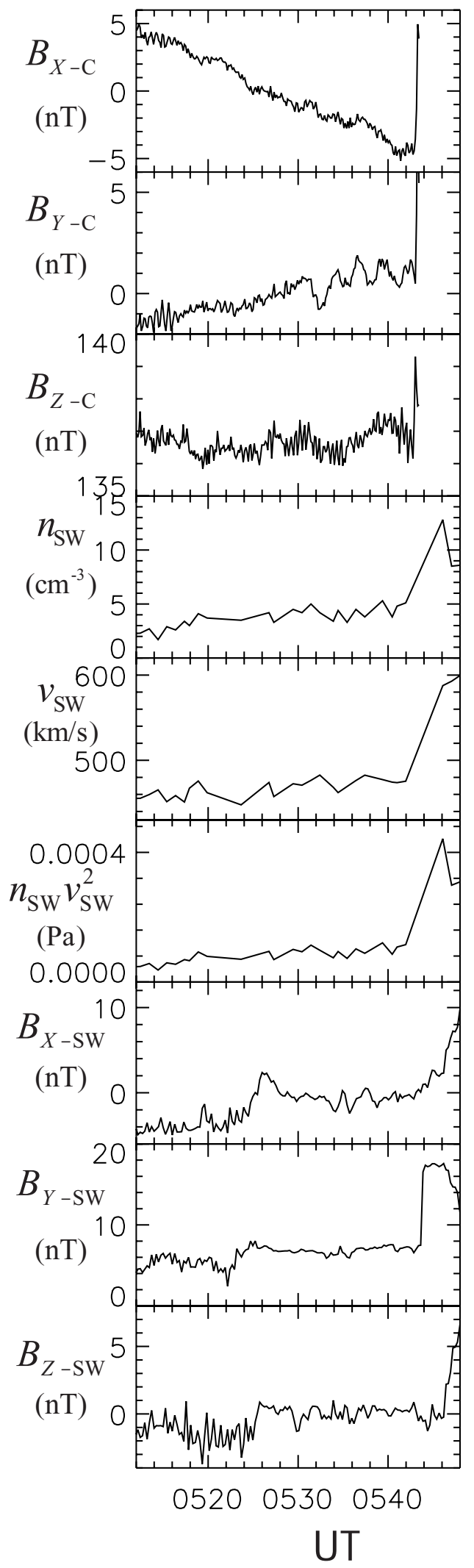

Fig. 9. Magnetic field at CRRES (top three panels) compared with IMP 8 measurements of solar wind (next six panels) density, velocity, dynamic pressure, and magnetic field in GSM coordinates for the time interval 05:10-05:50 UT, 26 August 1990. 


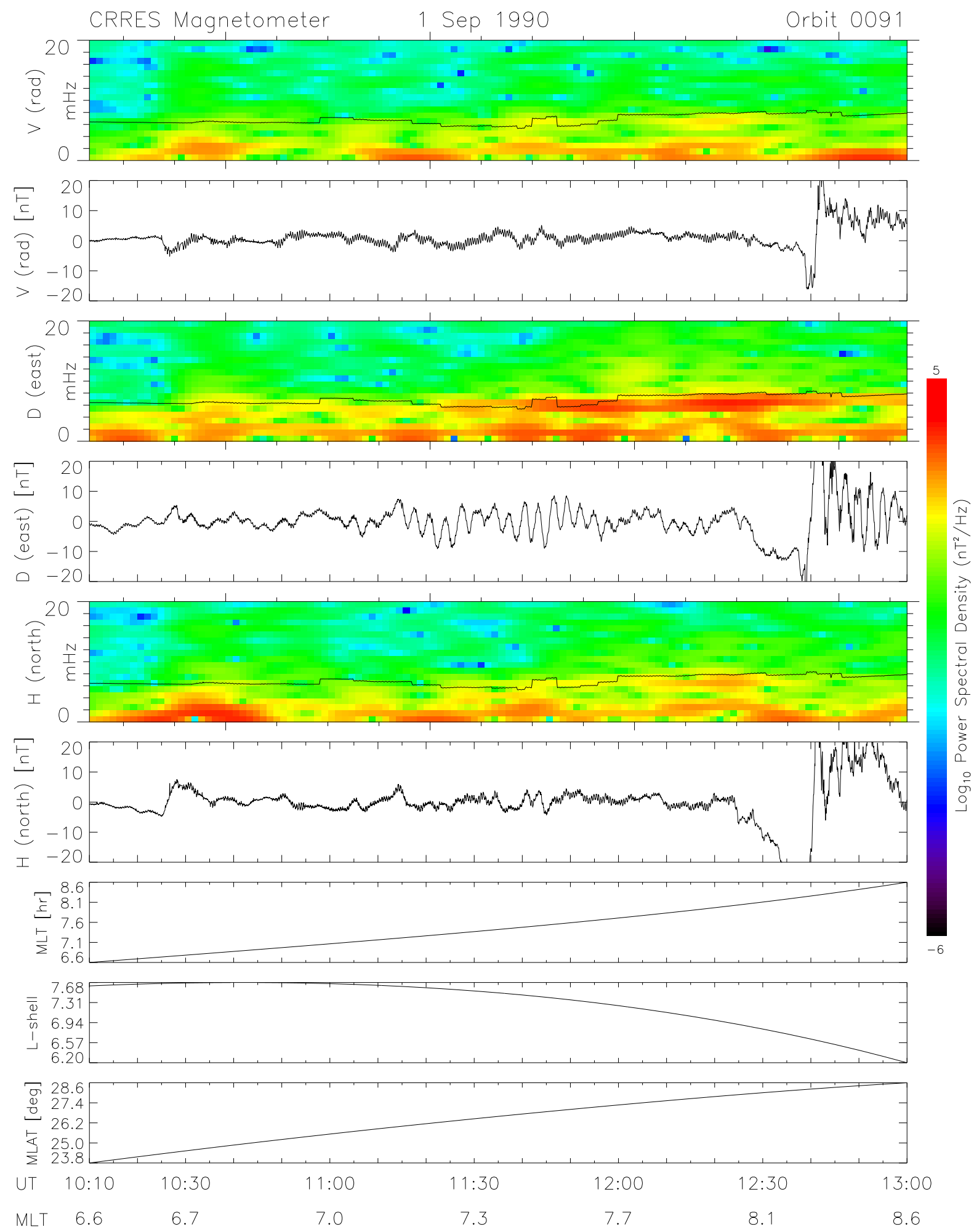

Fig. 10. SSC event of 1 September 1990, same format as Fig. 3. 
over all levels of geomagnetic activity (Fig. 4 for toroidal and Fig. 6 for poloidal modes). It is important to note that our statistical study is based on 2-4h intervals of data encompassing the SSCs, which allows for the buildup of the ring current. A study of the polarization characteristics of ULF waves associated with SSCs with a longer period than the fourteen month lifetime of CRRES will improve our confidence in the statistical significance of Fig. 7.

CRRES provides the only near-equatorial plane, geosynchronous-transfer orbit data to date on ULF wave mode structure and power in the inner magnetosphere. CRRES measurements covered a period of high geomagnetic activity at the Cycle 22 solar maximum, which may account for the greater dawn-dusk symmetry in toroidal mode power than reported in the AMPTE studies from solar minimum. Consistency with the GOES 6 study during the active six month interval from July until December 1993 (Glassmeier and Stellmacher, 2000) suggests that one should look for a greater longitudinal extent of in situ toroidal mode power in the more recent Cycle 23 solar maximum data. Unfortunately, there is no comparable near-equatorial plane coverage available for the most recent solar maximum, although the Polar satellite apogee passes through the geomagnetic equatorial plane in summer 2002, and may provide some complimentary data to that currently available from geosynchronous spacecraft. Also, there is now continuous solar wind data available from the Advanced Composition Explorer satellite located in a halo orbit about the L1 point, $230 R_{E}$ upstream from Earth, unlike the CRRES era when only one of the SSCs examined in the present study (26 August 1990) had good solar wind coverage by the Earth-orbiting IMP 8 spacecraft. Future studies will be able to address the question of directly driven oscillations in the magnetosphere, as Kepko et al. (2002) have recently shown in comparing WIND solar wind pressure measurements at L1 with GOES 10 geosynchronous magnetometer data. They conclude that discrete spectral peaks below $\sim 3 \mathrm{mHz}$ at geosynchronous orbit may be directly driven by solar wind pressure variations at the same frequencies, below fundamental FLR eigenfrequencies. Simultaneous measurement of the local plasma density and calculation of the fundamental and harmonic eigenfrequencies has enabled us to distinguish higher frequency ULF wave observations as discrete eigenmodes of the magnetosphere at and inside geosynchronous orbit using the CRRES data set.

Acknowledgements. We thank NSSDC for making IMP-8 (GSFC and LANL) data available on OMNIWeb and Howard Singer and Jeff Hughes for use of the CRRES magnetometer data. Work at Dartmouth was supported by NSF grant ATM-9911975 and NASA grant NAG5-11712 and NAG5-11735.

Topical Editor T. Pulkkinen thanks H. Singer and K. Yumoto for their help in evaluating this paper.

\section{References}

Anderson, B. J.: An overview of spacecraft observations of $10 \mathrm{~s}$ to $60 \mathrm{~s}$ period magnetic pulsations in the Earth's magnetosphere, in: Solar Wind sources of Ultra-Low-Frequency Waves, edited by Engebretson, M. J., Takahashi, K., and Scholer, M., AGU, Washington, D. C., 1994.

Anderson, B. J., Engebretson, M. J., Rounds, S. P., Zanettii, L. J., and Potemra, T. A.: A statistical study of Pc 3-5 pulsations observed by the AMPTE/CCE magnetic fields experiment: 1. Occurrence distribution, J. Geophys. Res., 95, 10 495, 1990.

Anderson, R. R., Gurnett, D. A., and Odem, D. L.: CRRES plasma wave experiment, J. Spacecraft and Rockets, 29, 570, 1992.

Chen, L., and Hasegawa, A.: A theory of long-period magnetic pulsation, 1, steady state excitation of field line resonance, J. Geophys, Res., 79, 1024, 1974.

Chen, L. and Hasegawa, A.: On magnetospheric hydromagnetic waves excited by energetic ring current particles, J. Geophys. Res., 93, 8763, 1988.

Chen, L. and Hasegawa, A.: Kinetic theory of geomagnetic pulsations, 1, Internal excitations by energetic particles, J. Geophys. Res., 96, 1503, 1991.

Clemmons, J. H., Pfaff, R. F., Lennartsson, O. W., Mozer, F. S., Singer, H. J., Peterson, W. K., Scudder, J. D., Kletzing, C. A., Chi, P. J., Wallis, D. D., and Larson, D. E.: Observations of travelling Pc5 waves and their relation to the magnetic cloud event of January 1997, J. Geophys. Res. - Space Phys., 105 (A3), 54415452, 2000.

Cheng, C. Z. and Lin, C. S.: Eigenmode analysis of compressional waves in the magnetosphere, J. Geophys. Res., 14, 884, 1987.

Cummings, W. D., O'Sullivan, R. J., Coleman, Jr., P. J.: Standing Alfvén Waves in the Magnetosphere, J. Geophys. Res., 74, 778, 1969.

Denton, R. E. and Gallagher, D. L.: Determining the mass density along magnetic field lines from toroidal eigenfrequencies, J. Geophys. Res., 105, 27 717, 2000.

Denton, R. E., Lessard, M. R., Anderson, R., Miftakhova, E. G., and Hughes, J. W.: Determining the mass density along magnetic field lines from toroidal eigenfrequencies: Polynomial expansion applied to CRRES data, J. Geophys. Res., 106, 29 915, 2001.

Dungey, J. W.: Electrodynamics of the outer atmospheres, Tech. Rep. 69, Ions. Res. Lab. Pa. State Univ., University Park, 1954.

Engebretson, M. J., Glassmeier, K.-H., Stellmacher, M., Hughes, W. J., and Luhr, H.: The dependence of high latitude Pc-5 wave power on solar wind velocity and on the phase of high speed solar wind streams, J. Geophys. Res., 103, 26271, 1998.

Fenrich, F. R., Samson, J. C., Sofko, G., and Greenwald, R. A.: ULF high and low-m field line resonances observed with SuperDARN, J. Geophys. Res., 100, 21 535, 1995.

Geomagnetic Data 1990, IAGA Bulletin no. 32u, 1996.

Geomagnetic Data 1991, IAGA Bulletin no. 32v, 1998.

Gallagher, D. L., Craven, P. D., and Comfort, R. H.: Global core plasma model, J. Geophys. Res., 105, 18 819, 2000.

Goldstein, J., Hudson, M. K., and Lotko, W.: Possible evidence of damped cavity mode oscillations stimulated by the January, 1997 magnetic cloud event, Geophys. Res. Lett., 26, 3589, 1999.

Glassmeier, K.-H.: ULF pulsations, in: Handbook of Atmospheric Electrodynamics, Vol. 2, edited by Volland, H., CRC Press, 463502, Boca Raton, 1995.

Glassmeier, K.-H. and Stellmacher, M.: Concerning the local time asymmetry of Pc-5 wave power at the ground and field line resonance widths, J. Geophys. Res., 105, 18 847, 2000. 
Holzer, T. E.: The solar wind and related astrophysical phenomena, in: Solar System Plasma Physics, V.1, 101-176, edited by Kennel, C. F., Lanzerotti, L. J., and Parker, E. N., North Holland Pub. Co., 1979.

Hughes, W. J.: Magnetospheric ULF waves: A tutorial with a historical perspective, in: Solar Wind sources of Ultra-Low- Frequency Waves, edited by Engebretson, M. J., Takahashi, K., and Scholer, M., AGU, Washington, D. C., 1994.

Jacobs, J. A., Kato, Y., Matsushita, S., and Troitskaya, V. A.: Classification of geomagnetic micropulsations, J. Geophys. Res., 69, 180,1964

Kepko, L., Spence, H. E., and Singer, H. E.: ULF waves in the solar wind as direct drivers of magnetospheric pulsations, J. Geophys. Res., 29, 39-1, 2002.

Knipp, D. J., Emery, B. A., Engebretson, M., Li, X., McAllister, A. H., Mukai, T., Kokubun, S., Reeves, G. D., Evans, D., Obara, T., Pi, X., Rosenberg, T., Weatherwax, A., McHarg, M. G., Chun, F., Mosely, K., Codrescu, M., Lanzerotti, L., Rich, F. J., Sharber, J., and Wilkinson, P.: An overview of the early November, 1993 geomagnetic storm, J. Geophys. Res. - Space Phys., 103 (A11),26 197-26220, 1998.

Lee, L. C., Albano, R. K., and Kan, J. R.: Kelvin-Helmholz instability in the magnetopause-boundary layer region, J. Geophys. Res., 86, 54, 1981.

Lessard, M., Hudson, M. K., and Luhr, H.: A statistical study of Pc3-Pc5 magnetic pulsations observed by the AMPTE/Ion Release Module satellite, J. Geophys. Res., 104, 4523, 1999.

Miura, A.: Kelvin-Helmholz Instability at the Magnetospheric Boundary: Dependence on the Magnetosheath Sonic Mach Number, J. Geophys. Res., 97, 10 655, 1992.

O'Brien, T. P., McPherron, R. L., Sornette, D., Reeves, G. D., Friedel, R., and Singer, H. J.: Which magnetic storms produce relativistic electrons at geosynchronous orbit?, J. Geophys. Res., 106, 15 553, 2001.

Radoski, H. R.: A note on oscillating field lines, J. Geophys. Res., 72, 418, 1967.

Samson, J. C. and Rostoker, G.: Latitude-dependent characteristics of high-latitude Pc-4 and Pc-5 micropulsations, J. Geophys. Res., 77, 6133, 1972.

Schulz, M.: Eigenfrequencies of geomagnetic field lines and implications for plasma-density modeling, J. Geophys. Res., 101,
$17385,1996$.

Singer, H. J., Sullivan, W. P., Anderson, P., Mozer, F., Harvey, P., Wygant, J., and McNeil, W.: Fluxgate magnetometer instrument on the CRRES satellite, J. Spacecraft and Rockets, 29, 4599, 1992.

Sonnerup, B., Cahill, L. J., and Davis, L. R.: Resonant vibration of the magnetosphere observed from Explorer 26, J. Geophys. Res., 74, 2276-2288, 1969.

Southwood, D. J.: Some features of field line resonances in the magnetosphere, Planet. Space Sci., 22, 483, 1974.

Southwood, D. J.: A general approach to low-frequency instability in the ring current plasma, J. Geophys. Res., 81, 3340, 1976.

Southwood, D. J ., Dungey, J. W., and Eherington, R. L.: Bounce resonant interaction between pulsations and trapped particles, Planet Space Sci., 17, 349-361, 1969.

Stewart, B.: On the great magnetic disturbance which extended from August 2 to September 7, 1859 as recorded by photography at the Kew Observatory, Phil. Trans. Roy. Soc. Lond., 11, 407, 1861.

Takahashi, K., Higbie, P., and Baker, D. N.: Azimuthal propagation and frequency characteristics of compressional Pc5 waves observed at geostationary orbit, J. Geophys. Res. - Space Phys., 90 (NA2), 1473-1485, 1985.

Takahashi, K., Denton, R. E., Gallagher, D.: Toroidal wave frequency at $\mathrm{L}=6-10$ : Active Magnetospheric Particles Tracer Explorers/CCE observations and comparison with theoretical model, J. Geophys. Res., 107, DOI10.1029/2001JA000197, 2002.

Vetoulis, G. and Chen, L.: Kinetic theory of geomagnetic pulsations, 3, Global analysis of drift Alfvén ballooning modes, J. Geophys. Res., 101, 15, 441, 1996.

Walker, A. D. M., Greenwald, R. A., Stuart, W. F., and Green, C. A.: STARE auroral radar observations of Pc-5 geomagnetic pulsations, J. Geophys. Res., 84, 3373, 1979.

Yumoto, K., Saito, T., and Sakurai, T.: Local time asymmetry in the characteristics of Pc-5 magnetic pulsations, Planet. Space Sci., 31, 459, 1983.

Zhu, X. and Kivelson, M. G.: Compressional ULF waves in the outer magnetosphere: 1. Statistical study, J. Geophys. Res., 96, $19451,1991$. 